\title{
Commentary Re: Botox Article by Berger \& Knoll IPH 19(5):243-244 on "The efficacy of botulinum toxin type A in managing chronic musculoskeletal pain: a systematic review and meta analysis"
}

\author{
Tony Zhang - Aleem Adatia - Wasifa Zarin - Misha Moitri - Abi Vijenthira • \\ Rong Chu $\cdot$ Lehana Thabane $\cdot$ Walter F. Kean
}

Received: 14 October 2011/Accepted: 2 November 2011/Published online: 15 November 2011

(C) Springer Basel AG 2011

While the quality assessment of RCTs in systematic reviews and meta-analyses are widely supported, the approach to doing so is not without debate. It is known that a wide range of scales and check-lists are employed in systematic reviews for assessing qualities of RCTs with Jadad scale being one of them (Juni et al. 2001; Olivo et al. 2008). We acknowledge that the scale is not without its flaws. However, Jadad scale is easy to apply and assess important attributes of a RCT, such as randomization, blinding, and attrition biases (Jadad et al. 1996; Juni et al. 2001). Furthermore, it has been developed on the basis of rigorous validity and reliability testing (Olivo et al. 2008). This is in contrast to other scales, most of which have not been appropriately evaluated (Olivo et al. 2008).

As alluded in the paper (Zhang et al. 2011), Jadad score only provides a general idea about the quality of a RCT. A score of 5 out of 5 does not certainly suggest that it is a perfect trial. Dr. Berger pointed out that the Wong et al. (2005) trial may have compromised its blinding protocol because the four patients who experienced paresis of

T. Zhang - A. Adatia

Schulich School of Medicine, University of Western Ontario, London, ON, Canada

W. Zarin · M. Moitri · A. Vijenthira

Faculty of Health Sciences, McMaster University,

Hamilton, ON, Canada

R. Chu $\cdot$ L. Thabane

Department of Epidemiology and Biostatistics,

McMaster University, Hamilton, ON, Canada

W. F. Kean ( $\square)$

Department of Medicine (Rheumatology), McMaster University,

401-1 Young Street, Hamilton, ON L8N 1T8, Canada

e-mail: keanmac@cogeco.ca fingers may have guessed that they received the botox treatment. However, this is only a conjecture. The only way to have known for sure was to ask the patients. Neither we nor the authors believe that this was a flaw significant enough to invalidate the results of the trial.

The purpose of our report is to provide a review of existing RCTs. In keeping with that goal, we provided detailed and transparent information about each trial, including individual Jadad score. While we share Dr. Berger's general concern over the quality assessment of RCTs, exhaustive scrutiny of every detail concerning a trial's methodology is often not possible.

\section{References}

Jadad AR, Moore RA, Carroll D et al (1996) Assessing the quality of reports of randomised controlled trials: is blinding necessary? Control Clin Trials 17:1-12

Juni P, Altman DG, Egger M (2001) Assessing the quality of randomized controlled trials. In: Egger M, Smith GD, Altman DG (eds) Systematic reviews in health care. Meta-analysis in context. BMJ Publishing Group, London, pp 87-109

Olivo SA, Macedo LG, Gadotti IC et al (2008) Scales to assess the quality of randomized controlled trials: a systematic review. Phys Ther 88(2):156-175

Wong SM, Hui ACF, Tong P-Y, Poon DWF et al (2005) Treatment of lateral epicondylitis with botulinum toxin. Ann Intern Med 143:793-797

Zhang T, Adatia A, Zarin W et al (2011) The efficacy of botulinum toxin type $\mathrm{A}$ in managing chronic musculoskeletal pain: a systematic review and meta-analysis. Inflammopharmacology 19(1):21-34 